\section{Acute obstructive hydrocephalus after subarachnoid haemorrhage}

Neurological deterioration within the first week after subarachnoid haemorrhage may be due to a haematoma, rebleed, or vasospasm and ischaemia. Although communicating hydrocephalus occurring within the first week may also contribute to deterioration, ${ }^{1-3}$ only rarely has acute obstructive (non-communicating) hydrocephalus been reported as a cause. ${ }^{4}$ We describe two cases in which this complication occurred within 12 hours after subarachnoid haemorrhage.

\section{Case reports}

Case 1-A 64 year old woman was admitted to a medical ward a few hours after sudden onset of severe headache associated with photophobia and neck stiffness. She was orientated and had no neurological deficit. Subarachnoid haemorrhage was confirmed by lumbar puncture. Two hours after admission her level of consciousness deteriorated slightly and she was subsequently transferred to our neurosurgical unit. On admission she was able to localise to a painful stimulus but did not open her eyes to speech and gave no verbal response. Emergency CT scan showed an acute obstructive hydrocephalus due to blood within the third ventricle (see figure). Five hours after the episode of subarachnoid haemorrhage a ventricular drain was inserted through a right frontal burr hole. Four hours later her level of consciousness had improved; she obeyed commands, gave a confused verbal response, and opened her eyes to speech. She later required a ventriculoperitoneal shunt.

Case 2-A 54 year old woman was awakened by severe occipital headache and vomiting. Later that day she was admitted to a general surgical ward, at which time she was obeying commands, talking, and opening her eyes to
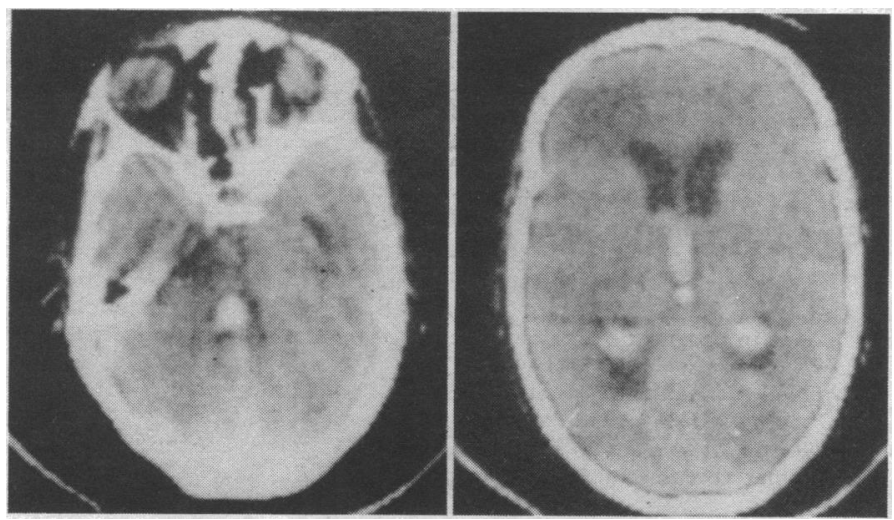

Case 2. CT scan of patient with acute obstructive hydrocephalus showing blood in all four ventricles.

speech. Lumbar puncture confirmed a subarachnoid haemorrhage. Her level of consciousness gradually deteriorated over eight hours and she was transferred to our unit. On admission she was able to localise to a painful stimulus but did not open her eyes to speech and gave no verbal response. A CT scan 12 hours after the initial episode showed an acute obstructive hydrocephalus with blood in all four ventricles (figure). Bifrontal ventricular drains were immediately inserted, and within two hours her level of consciousness had improved to obeying commands, giving a confused verbal response, and opening eyes to speech.

\section{Comment}

Sudden neurological deterioration in the first week after a subarachnoid haemorrhage may be due to either a rebleed or raised intracranial pressure caused by an expanding haematoma or associated brain swelling. Neurological deterioration progressing more slowly may be due to delayed ischaemia or development of communicating hydrocephalus. We describe acute obstructive hydrocephalus as a further cause of deterioration in two patients. Obstructive hydrocephalus is a reasonable explanation for the deterioration in consciousness in these patients because they improved soon after ventricular drainage. The role of the CT scan in the diagnosis and management of subarachnoid haemorrhage is well established. ${ }^{5}$ How common this readily treatable cause of progressive neurological deterioration is in subarachnoid haemorrhage is unknown. Increased use of the CT scan in the primary diagnosis of subarachnoid haemorrhage is likely to provide further information. Ventricular drainage may contribute to the early management of this condition.

We thank Mr T A H Hide for permission to report on his patients.

${ }^{1}$ Raimondi AJ, Torres H. Acute hydrocephalus as a complication of subarachnoid hemorrhage. Surg Neurol 1973;1:23-6.

2 Vassilouthis J, Richardson AE. Ventricular dilatation and communicating hydrocephalus following spontaneous subarachnoid hemorrhage. $\mathcal{F}$ Neurosurg 1979;51:341-51.

${ }^{3}$ Doczi T, Menessanji Z, Szegvary Z, Huszka E. Disturbances of cerebrospinal fluid circulation during the acute stage of subarachnoid hemorrhage. Neurosurgery 1983;12:435-8.

4 Kusske JA, Turner PR, Ojemann GA, Harris AB. Ventriculostomy for the treatment of acute hydrocephalus following subarachnoid hemorrhage. f Neurosurg 1975;38:591-5.

5 Kendell BE, Claveria E. Computerised tomography in subarachnoid haemorrhage. $B r \mathcal{~}$ Radiol 1976;49:483-501.

(Accepted 25 fanuary 1984)

Institute of Neurological Sciences, Southern General Hospital, Glasgow G51 4TF

Y M KOHI, FRCS, senior registrar

R A JOHNSTON, MD, FRCs, senior registrar

U P DEVKOTA, MB, BS, registrar

Correspondence to: $\mathrm{Mr}$ Y M Kohi.

\section{Response of urinary albumin to submaximal exercise in newly diagnosed non-insulin dependent diabetes}

Subtle changes in urinary albumin excretion are the first clinical indication of renal disease in diabetes mellitus and are thought to be caused by the excessive glycosylation of glomerular proteins. Exercise increases pathological albuminuria, and the response to standardised submaximum exercise provides a highly sensitive test of early glomerular dysfunction in diabetes. ${ }^{1}$ Thus albumin excretion remains unchanged in healthy people but may rise severalfold in clinically established insulin dependent diabetes. ${ }^{12}$ Screening studies suggest that non-insulin dependent diabetes is often preceded by a long period of asymptomatic hyperglycaemia, and the aim of our investigation in patients with newly diagnosed non-insulin dependent diabetes was to establish whether renal dysfunction was already established by the time the diabetes declared itself clinically.

\section{Patients, methods, and results}

The albuminuric response to submaximal exercise was examined in 10 newly diagnosed and untreated patients with non-insulin dependent diabetes (six men and four women aged 45-70) and seven healthy controls. A diuresis was first established by having the subjects consume $250 \mathrm{ml}$ of tap water every 20 minutes for 90 minutes before and during the test. Urine was collected over periods of 20 minutes immediately before and five minutes after a 10 minute exercise period on a treadmill. The heart rate on the treadmill was maintained between 100 and 130 beats/min in the men and 130-165 beats/ $\mathrm{min}$ in the women, equivalent to the $600 \mathrm{kpm} / \mathrm{min}$ bicycle stress test used by Mogensen and Vittinghus when studying albuminuria in insulin dependent diabetes. ${ }^{1}$ The test was performed before treatment and repeated four and eight weeks after the introduction of a high fibre, energy controlled diet.

Albumin was measured by a single sandwich enzyme linked immunosorbent assay with a coefficient of variation of less than $10 \%$ (Mohamed A, unpublished study). Capillary glucose was measured by BM Stix (Boehringer) 
and expressed as the mean of a seven point profile obtained over 18 hours before and once a week during the eight week period. Glycosylated haemoglobin $\left(\mathrm{HbA}_{1}\right)$ was measured by a commercial column method (Bio-Rad Laboratories) at the beginning and end of the study.

After dietary treatment the mean glycosylated haemoglobin of the patients fell from $12 \cdot 1$ (SD $3 \cdot 8) \%$ to $9.3(1 \cdot 1) \%(p<0.01$ ) (controls $7 \cdot 0(0.5) \%$ ). The mean capillary glucose concentration similarly fell from $8.6(0.8) \mathrm{mmol} / \mathrm{l}$ to $6.4(0.4) \mathrm{mmol}(p<0.01)$. The figure shows the urinary albumin excretion during the same period. At diagnosis the mean excretion rate was higher in

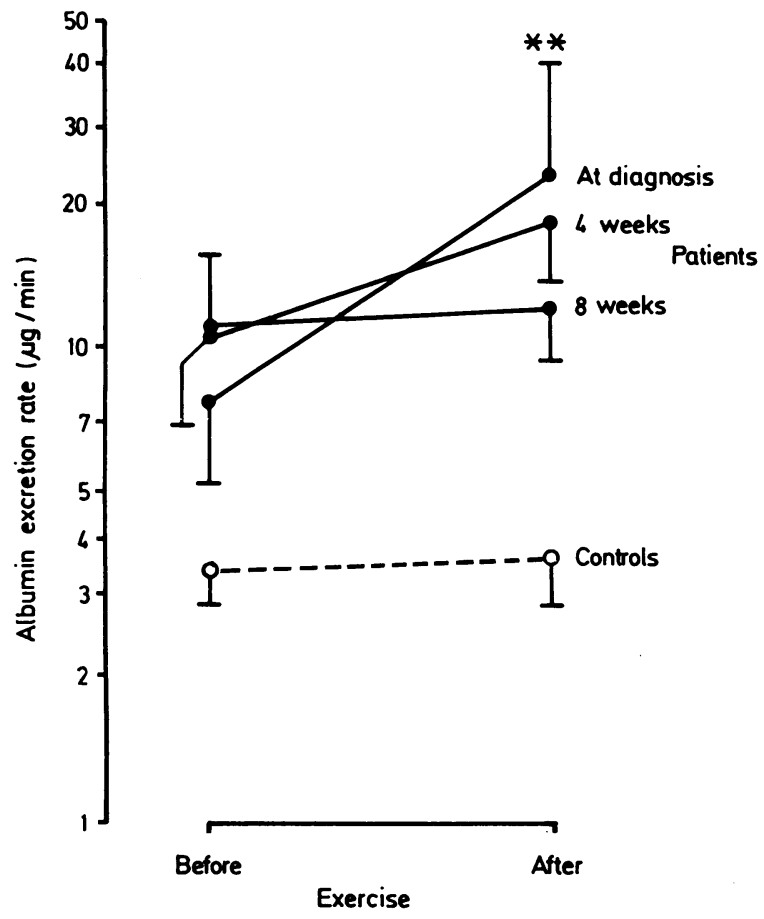

Mean albumin excretion rates before and after exercise in 10 newly diagnosed non-insulin dependent diabetics before (at diagnosis) and four and eight weeks after the introduction of dietary treatment, and in seven controls $(* *=p<0.01)$.

diabetics and the response to exercise statistically highly significant. With dietary treatment (and lowering of capillary glucose concentration and glycosylation index) the response of albuminuria to exercise fell progressively to zero, although the basal excretion rate remained unchanged.

\section{Comment}

These observations in non-insulin dependent diabetics suggest that their albumin excretion rate is rather higher at the time of diagnosis than that of controls and that, unlike that of controls, it increases substantially on submaximal exercise. Such a response is pathological, and we must consider seriously the suggestion from studies in insulin dependent diabetics that what starts as a functional abnormality of the glomerulus may progress if unchecked to irreversible renal failure. ${ }^{3}$ Indeed, recent evidence suggests that subclinically abnormal albumin excretion is a strong predictor of mortality in non-insulin dependent diabetes (Viberti et al, British Diabetic Association meeting, Norwich, 1983). Our preliminary experience suggests that dietary treatment alone may be sufficient to reverse the early anomaly.

Our finding that urinary albumin excretion was already abnormal at diagnosis in non-insulin dependent diabetics raises questions about how long glomerular dysfunction may be present before other manifestations of diabetes take the patient to the doctor and whether screening for non-insulin dependent diabetes may prove a fruitful public health prevention measure.

TJW is supported by the Wellcome Trust. Our thanks to Dr B Leppard for her kind cooperation and to Mrs I Strachan for secretarial help.

${ }^{1}$ Mogensen CE, Vittinghus E. Urinary albumin excretion during exercise in juvenile diabetes. Scan F Clin Lab Invest 1975;35:295-300.

2 Viberti GC, Pickup JC, Bilous RW, Keen H, McIntosh D. Correction of exercise-induced microalbuminuria in insulin dependent diabetics after 3 weeks of subcutaneous insulin infusion. Diabetes $1981 ; 30: 818-22$.
${ }^{3}$ Keen $\mathrm{H}$, Viberti GC. Genesis and evolution of diabetic nephropathy. $\mathcal{f}$ Clin Pathol $1981 ; 34: 1261-5$.

(Accepted 16 February 1984)

Departments of Medicine II and Chemical Pathology, General Hospital and Diabetes Clinic, Royal South Hants Hospital, Southampton SO9 4XY

A MOHAMED, medical student

TERENCE WILKIN, MRCP, Wellcome senior lecturer in endocrinology

B A LEATHERDALE, MRCP, consultant physician

D ROWE, PHD, top grade biochemist

Correspondence to: Dr Terence Wilkin, Professorial Medical Unit, Southampton General Hospital, Southampton SO9 4XY.

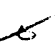

\section{Spinal cord disease due to Schistosoma mansoni successfully treated with oxamniquine}

Spinal cord disease is a rare manifestation of schistosomiasis and is usually caused by Schistosoma mansoni or $S$ haematobium. ${ }^{1}$ The diagnosis has typically been made at surgery or necropsy, and hitherto the most effective treatment for patients who developed sphincter problems was decompressive surgery. ${ }^{2}$ We describe a patient with $S$ mansoni infection who presented with symptoms of a spinal cord lesion and was rapidly and successfully treated with oxamniquine.

\section{Case report}

A 25 year old Ghanaian woman was admitted with acute retention of urine, having had difficulty passing urine for four days. Ten days before admission she had developed pain in the lumbar region and pains in her feet which steadily advanced up the legs. She had last been in Ghana three

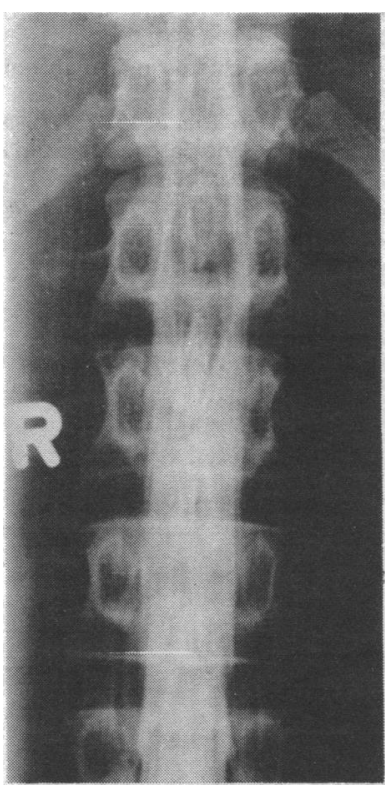

Myelogram showing expansion of conus medullaris, consistent with intramedullary mass. and a half years previously. Examination showed a healthy looking black woman with no fever or enlargement of abdominal organs. Power, deep tendon and superficial reflexes, and plantar responses were normal. There was no sensory deficit in the legs or sacral area.

Investigations disclosed a normal haemoglobin concentration and erythrocyte sedimentation rate. The leucocyte count was $6.4 \times 10^{\circ} / 1$, with $14 \%$ eosinophils $\left(890 \times 10^{6} / 1\right)$. The myelogram showed considerable expansion of the conus medullaris in both the anteroposterior and lateral views at the level of the first lumbar vertebra (figure), with appearances consistent with an intramedullary mass. Cerebrospinal fluid contained protein $0.57 \mathrm{~g} / 1$, glucose $0.02 \mathrm{mmol} / 1(0.34 \mathrm{mg} / 100 \mathrm{ml})$ (blood glucose concentration 0.03 $\mathrm{mmol} / 1 ; 0.62 \mathrm{mg} / 100 \mathrm{ml}$ ), and a white cell count of $66 \times 10^{9} / 1(95 \%$ lymphocytes). Cerebrospinal fluid IgG was raised at $0.26 \mathrm{~g} / 1$, and electrophoresis showed a diffuse increase in gammaglobulin. Rectal biopsy yielded the dead ova of $S$ mansoni. No ova were found on repeated examination of stools and urine. Schistosomal enzyme linked immunosorbent assay antibody titre was raised at 1.5 times the upper screening level.

Despite repeated attempts to remove the catheter fitted on admission this could not be done until two weeks later, when the patient was given oxamniquine $30 \mathrm{mg} / \mathrm{kg}$ by mouth for two days. Within 72 hours of starting treatment she was able to pass urine without difficulty and the pains in her legs almost completely cleared. She suffered no side effects. At follow up six months later she remained perfectly well with no recurrence of symptoms.

\section{Comment}

This patient's illness was typical of schistosomiasis affecting the spinal cord, with pains in the legs and lower back, accompanied by sphincter disturbance. The diagnosis was suspected on the basis of 